Miklós Laczkovich* Department of Analysis, Eötvös Loránd University, Rákóczi út 5, Budapest, Hungary 1088. e-mail: laczk@cs.elte.hu

\title{
TWO CONSTRUCTIONS OF SIERPIŃSKI AND SOME CARDINAL INVARIANTS OF IDEALS
}

\begin{abstract}
Let $\mathcal{N}$ denote the ideal of null sets in $\mathbb{R}$. Using $\mathrm{CH}$ Sierpiński constructed a set $A \subset \mathbb{R}$ satisfying $A \notin \mathcal{N}, \mathbb{R} \backslash A \notin \mathcal{N}$, and $(A+h) \backslash A \in \mathcal{N}$ for every $h \in \mathbb{R}$. He also constructed a set $H \subset \mathbb{R}^{2}$ such that each horizontal section of $H$ and the complement of each vertical section of $H$ belong to $\mathcal{N}$. In this note we investigate the existence of sets with analogous properties when $\mathcal{N}$ is replaced by an arbitrary invariant ideal. We also discuss the relationship among several related statements, including some inequalities between the cardinal invariants of the ideal in question.

In the case of null sets in $\mathbb{R}$ we show that the nonexistence of $A \subset \mathbb{R}$ with $A \notin \mathcal{N}, \mathbb{R} \backslash A \notin \mathcal{N}$, and $(A+h) \backslash A \in \mathcal{N}(h \in \mathbb{R})$ is equivalent to the difference property of the class $\mathcal{L}$ of Lebesgue measurable functions defined on $\mathbb{R}$. As an application we obtain that the difference property of the class $\mathcal{L}$ is consistent with ZFC.
\end{abstract}

\section{The Diagram.}

It is well-known that the following two statements are consequences of $\mathrm{CH}$.

$S^{1}:$ There exists a set $A \subset \mathbb{R}$ such that both $A$ and $\mathbb{R} \backslash A$ are of positive outer measure, but $(A+h) \backslash A$ is of measure zero for every $h \in \mathbb{R}$.

$S^{2}:$ There exists a set $H \subset \mathbb{R}^{2}$ such that the horizontal section $H^{y}=\{x$ : $(x, y) \in H\}$ is of measure zero for every $y \in \mathbb{R}$, and the complement of the vertical section $H_{x}=\{y:(x, y) \in H\}$ is of measure zero for every $x \in \mathbb{R}$.

\footnotetext{
Mathematical Reviews subject classification: 03E35, 03E65, 04A20

Received by the editors July 27, 1998

* Supported by the Hungarian National Foundation for Scientific Research, Grant T016094
} 
(See [11], Propositions $C_{70}$ and $C_{49}$. See also [9] and [10].) In this note we discuss the relationships between $S^{1}, S^{2}$, and several other statements involving certain cardinal numbers. In fact, we shall consider these relationships in a more general setting, when $\mathbb{R}$ is replaced by an arbitrary Abelian group $X$ and the system of Lebesgue null sets is replaced by a translation-invariant ideal of subsets of $X$.

Let $\mathcal{I}$ be an ideal of sets. (For the basic facts concerning ideals and cardinal invariants of ideals, the reader can consult [1].) We shall always assume that $\mathcal{I}$ is nontrivial in that $\mathcal{I} \neq \emptyset$ and $\bigcup \mathcal{I} \notin \mathcal{I}$. The cardinality of a set $H$ will be denoted by $|H|$. We shall need the following cardinal functions of $\mathcal{I}$.

$$
\begin{aligned}
& \operatorname{add}(\mathcal{I})=\min \{|\mathcal{H}|: \mathcal{H} \subset \mathcal{I}, \cup \mathcal{H} \notin \mathcal{I}\}, \\
& \operatorname{cov}(\mathcal{I})=\min \{|\mathcal{H}|: \mathcal{H} \subset \mathcal{I}, \cup \mathcal{H}=\cup \mathcal{I}\}, \\
& \operatorname{non}(\mathcal{I})=\min \{|E|: E \subset \cup \mathcal{I}, E \notin \mathcal{I}\}, \\
& \operatorname{cof}(\mathcal{I})=\min \{|\mathcal{H}|: \mathcal{H} \subset \mathcal{I}, \forall E \in \mathcal{I} \exists H \in \mathcal{H}, E \subset H\}, \\
& \operatorname{non}^{*}(\mathcal{I})=\min \{\kappa: \forall E \notin \mathcal{I} \exists F \subset E \text { such that } F \notin \mathcal{I} \text { and }|F| \leq \kappa\} .
\end{aligned}
$$

In order to make the diagram of the next proposition symmetric, we shall introduce one more cardinal (the "chain cover" of $\mathcal{I}$ ). We say that $\mathcal{H} \subset \mathcal{I}$ is a chain if, for every $A, B \in \mathcal{H}$ we have either $A \subset B$ or $B \subset A$. Let $\operatorname{ccv}(\mathcal{I})$ denote the smallest cardinal $\kappa$ such that for every chain $\mathcal{H} \subset \mathcal{I}$ there is $\mathcal{F} \subset \mathcal{I}$ such that $\bigcup \mathcal{H} \subset \bigcup \mathcal{F}$ and $|\mathcal{F}| \leq \kappa$.

Proposition 1. In the following diagram the cardinals increase from southwest to north-east.

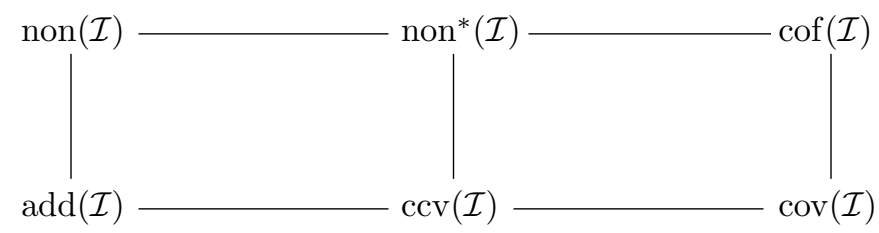

Diagram 1.

In other words, $\operatorname{add}(\mathcal{I}) \leq \operatorname{non}(\mathcal{I}) \leq \operatorname{non}^{*}(\mathcal{I}) \leq \operatorname{cof}(\mathcal{I}), \operatorname{add}(\mathcal{I}) \leq \operatorname{ccv}(\mathcal{I}) \leq$ $\operatorname{cov}(\mathcal{I}) \leq \operatorname{cof}(\mathcal{I})$, and $\operatorname{ccv}(\mathcal{I}) \leq \operatorname{non}^{*}(\mathcal{I})$.

Proof. The inequalities $\operatorname{add}(\mathcal{I}) \leq \operatorname{non}(\mathcal{I}) \leq \operatorname{non}^{*}(\mathcal{I})$ and $\operatorname{ccv}(\mathcal{I}) \leq \operatorname{cov}(\mathcal{I}) \leq$ $\operatorname{cof}(\mathcal{I})$ are obvious. 
$\operatorname{non}^{*}(\mathcal{I}) \leq \operatorname{cof}(\mathcal{I}):$ Let $\operatorname{cof}(\mathcal{I})=\kappa$, and select $\mathcal{H} \subset \mathcal{I}$ such that $|\mathcal{H}|=\kappa$ and for every $N \in \mathcal{I}$ there is an $H \in \mathcal{H}$ with $N \subset H$. If $E \notin \mathcal{I}$, then $E \backslash H \neq \emptyset$ for every $H \in \mathcal{H}$. Let a point $x_{H} \in E \backslash H$ be selected for every $H \in \mathcal{H}$, and put $F=\left\{x_{H}: H \in \mathcal{H}\right\}$. Then $F \subset E$ and $F \notin \mathcal{I}$, since $F$ is not covered by any element of $\mathcal{H}$. This implies $|F| \leq|\mathcal{H}|=\kappa$, which proves non* $(\mathcal{I}) \leq \kappa$.

$\operatorname{add}(\mathcal{I}) \leq \operatorname{ccv}(\mathcal{I}): \operatorname{Let} \operatorname{add}(\mathcal{I})=\kappa$, and select $\mathcal{F} \subset \mathcal{I}$ such that $|\mathcal{F}|=\kappa$, and $\bigcup \mathcal{F} \notin \mathcal{I}$. Let $\left\{E_{\alpha}: \alpha<\kappa\right\}$ be a well-ordering of $\mathcal{F}$, and put $\mathcal{H}=$ $\left\{\bigcup_{\beta<\alpha} E_{\beta}: \alpha<\kappa\right\}$. Then $\mathcal{H}$ is a chain, $\mathcal{H} \subset \mathcal{I}$, and $\bigcup \mathcal{H} \notin \mathcal{I}$. By the definition of $\kappa, \cup \mathcal{H}$ is not covered by less than $\kappa$ elements of $\mathcal{I}$. Therefore, $\operatorname{ccv}(\mathcal{I}) \geq \kappa$.

$\operatorname{ccv}(\mathcal{I}) \leq \operatorname{non}^{*}(\mathcal{I}):$ Let non* $(\mathcal{I})=\kappa$. We have to prove that for every chain $\mathcal{H} \subset \mathcal{I}$ there is $\mathcal{F} \subset \mathcal{I}$ such that $\bigcup \mathcal{H} \subset \bigcup \mathcal{F}$ and $|\mathcal{F}| \leq \kappa$. Let $\bigcup \mathcal{H}=D$. If $D \in \mathcal{I}$, then $\mathcal{F}=\{D\}$ satisfies the requirements. Therefore we may assume $D \notin \mathcal{I}$. Then there is a set $F \subset D$ such that $F \notin \mathcal{I}$ and $|F| \leq \kappa$. For every $x \in F$ select an $H_{x} \in \mathcal{H}$ with $x \in H_{x}$, and put $\mathcal{F}=\left\{H_{x}: x \in F\right\}$. Then $\mathcal{F} \subset \mathcal{H}$ and $|\mathcal{F}| \leq \kappa$. We prove $\bigcup \mathcal{F}=D$. Suppose this is not true, and let a point $y \in D \backslash \bigcup \mathcal{F}$ be selected. If $H \in \mathcal{H}$ contains $y$, then $H_{x} \subset H$ for every $x \in F$, since $\mathcal{H}$ is a chain, and $H \subset H_{x}$ is impossible because $y \notin H_{x}$. Thus $F \subset \bigcup \mathcal{F} \subset H \in \mathcal{I}$, contrary to $F \notin \mathcal{I}$.

Let $X$ be an Abelian group, and let $\mathcal{I}$ be an ideal of subsets of $X$. We shall use the notation $\mathcal{I} \mid A=\{B \subset A: B \in \mathcal{I}\}$ for every $A \subset X$. The ideal $\mathcal{I}$ is called invariant, if $E \in \mathcal{I}$ implies $E+x \in \mathcal{I}$ for every $x \in X$.

We shall investigate the relationship between the following statements.

$C_{\mathcal{I}}: X$ is the union of a chain of sets belonging to $\mathcal{I}$. (It is easy to see that $C_{\mathcal{I}}$ is equivalent to the existence of an ordering $\prec$ of $X$ such that each initial segment of $X$ belongs to $\mathcal{I}$; that is, $\{y: y \prec x\} \in \mathcal{I}$ for every $x$.)

$G_{\mathcal{I}}: X$ is the union of a chain of subgroups belonging to $\mathcal{I}$.

$S_{\mathcal{I}}^{1}:$ There exists a set $A \subset X$ such that $A \notin \mathcal{I}, X \backslash A \notin \mathcal{I}$ and $(A+h) \backslash A \in \mathcal{I}$ for every $h \in X$.

$S_{\mathcal{I}}^{1 s}:$ There exists a function $f: X \rightarrow X$ such that $\{x \in X: f(x+h)-f(x) \neq$ $0\} \in \mathcal{I}$ for every $h \in X$, and $f^{-1}(\{y\}) \in \mathcal{I}$ for every $y \in X$.

$S_{\mathcal{I}}^{2}$ : There exists a set $H \subset X \times X$ such that $H^{y} \in \mathcal{I}$ for every $y \in X$, and $X \backslash H_{x} \in \mathcal{I}$ for every $x \in X$.

$S_{\mathcal{I}}^{2 w}:$ There exists a set $H \subset X \times X$ such that $H^{y} \in \mathcal{I}$ for every $y \in X$, and $\left\{x: X \backslash H_{x} \in \mathcal{I}\right\} \notin \mathcal{I}$. 
$D_{\mathcal{I}}:$ There exists a decomposition $X=A \cup B$ such that $\operatorname{cov}(\mathcal{I} \mid A) \leq \operatorname{non}(\mathcal{I} \mid B)$ and $\operatorname{cov}(\mathcal{I} \mid B) \leq \operatorname{non}(\mathcal{I} \mid A)$.

Theorem 2. Let $X$ be an uncountable Abelian group and let $\mathcal{I}$ be an invariant ideal in $X$. Then each implication of the following diagram holds, except perhaps $S_{\mathcal{I}}^{1 s} \Longrightarrow S_{\mathcal{I}}^{1}$. This implication is also true, supposing that $\mathcal{I}$ is a $\sigma$-ideal and $|X|$ is less than the first (2-valued) measurable cardinal.

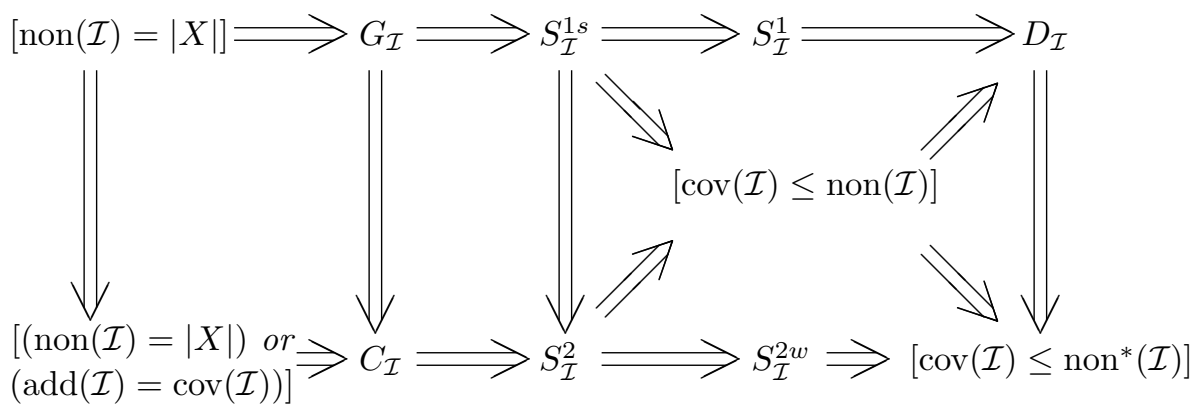

Diagram 2.

Proof. First we prove the implications of the first row.

$\left[[\operatorname{non}(\mathcal{I})=|X|] \Longrightarrow G_{\mathcal{I}}:\right.$ Let $\left\{x_{\alpha}: \alpha<|X|\right\}$ be a well-ordering of $X$, and let $G_{\alpha}$ denote the subgroup generated by $\left\{x_{\beta}: \beta<\alpha\right\}$. Since $\left|G_{\alpha}\right|=$ $\max (|\alpha|, \omega)<|X|$, it follows from non $(\mathcal{I})=|X|$ that $G_{\alpha} \in \mathcal{I}$ for every $\alpha$. Then $\left\{G_{\alpha}: \alpha<|X|\right\}$ is a chain of groups belonging to $\mathcal{I}$ whose union is $X$.

$G_{\mathcal{I}} \Longrightarrow S_{\mathcal{I}}^{1 s}$ : Let $\left\{G_{\alpha}: \alpha<\kappa\right\}$ be a chain of groups belonging to $\mathcal{I}$ such that $\cup_{\alpha<\kappa} G_{\alpha}=X$. Let $\Gamma$ denote the set of those ordinals $\alpha<\kappa$ for which the set $G_{\alpha}^{\prime}=G_{\alpha} \backslash \cup_{\beta<\alpha} G_{\beta}$ is nonempty, and select a point $y_{\alpha} \in G_{\alpha}^{\prime}$ for every $\alpha \in \Gamma$.

We define $f(x)=y_{\alpha}$, where $\alpha=\alpha(x)$ is the smallest ordinal with $x \in G_{\alpha}$. Then we have $f^{-1}\left(\left\{y_{\alpha}\right\}\right) \subset G_{\alpha} \in \mathcal{I}$ for every $\alpha \in \Gamma$. Let $h \in X$ be fixed. If $h \in G_{\alpha}^{\prime}$ and $x \notin G_{\alpha}$, then $G_{\beta} \subset G_{\alpha}$ for every $\beta<\alpha$, and thus $x \in G_{\gamma}^{\prime}$, where $\alpha<\gamma$ and $G_{\alpha} \subset G_{\gamma}$. Therefore $f(x+h)=f(x)=y_{\gamma}$, which proves $\{x: f(x+h)-f(x) \neq 0\} \subset G_{\alpha} \in \mathcal{I}$.

$S_{\mathcal{I}}^{1 s} \Longrightarrow S_{\mathcal{I}}^{1}$ (supposing that $\mathcal{I}$ is a $\sigma$-ideal and $|X|$ is less than the first measurable cardinal): Let $f$ be as in $S_{\mathcal{I}}^{1 s}$. Let $\mathcal{J}=\left\{H \subset X: f^{-1}(H) \in \mathcal{I}\right\}$; then $\mathcal{J}$ is a $\sigma$-ideal on $X$ containing the singletons. Since $|X|$ is less than the first measurable cardinal, $\mathcal{J}$ cannot be prime. That is, there exists an $H \subset X$ 
such that $f^{-1}(H) \notin \mathcal{I}$ and $X \backslash f^{-1}(H) \notin \mathcal{I}$. It is easy to check that the set $A=f^{-1}(H)$ satisfies the requirements of $S_{\mathcal{I}}^{1}$.

$S_{\mathcal{I}}^{1} \Longrightarrow D_{\mathcal{I}}$ : Let $A$ satisfy $S_{\mathcal{I}}^{1}$. We show that $\operatorname{cov}(\mathcal{I} \mid A) \leq \operatorname{non}(\mathcal{I} \mid(X \backslash A))$ and $\operatorname{cov}(\mathcal{I} \mid(X \backslash A)) \leq \operatorname{non}(\mathcal{I} \mid A)$. By symmetry it is enough to prove the first inequality. Let $\operatorname{non}(\mathcal{I} \mid(X \backslash A))=\kappa$, and select a set $B \subset X \backslash A$ such that $|B|=\kappa$, and $B \notin \mathcal{I}$. Since $(A-h) \backslash A \in \mathcal{I}$, we have $B \not \subset A-h$ for every $h$. This gives $\bigcap\{A-b: b \in B\}=\emptyset$, since $h \in A-b(b \in B)$ would imply $B \subset A-h$. Therefore $A=\bigcup\{A \backslash(A-b): b \in B\}$; that is, $A$ can be covered by $\kappa=\operatorname{non}(\mathcal{I} \mid(X \backslash A))$ elements of $\mathcal{I}$.

Next we prove the vertical implications. The first two relations are obvious. $S_{\mathcal{I}}^{2}$

$S_{\mathcal{I}}^{1 s} \Longrightarrow S_{\mathcal{I}}^{2}$ : If $f$ satisfies $S_{\mathcal{I}}^{1 s}$, then $H=\{(x, y): f(x+y) \neq f(x)\}$ satisfies

$D_{\mathcal{I}} \Longrightarrow\left[\operatorname{cov}(\mathcal{I}) \leq \operatorname{non}^{*}(\mathcal{I})\right]:$ Suppose $D_{\mathcal{I}}$. Then

$\operatorname{cov}(\mathcal{I}) \leq \max (\operatorname{cov}(\mathcal{I} \mid A), \operatorname{cov}(\mathcal{I} \mid B)) \leq \max (\operatorname{non}(\mathcal{I} \mid B), \operatorname{non}(\mathcal{I} \mid A)) \leq \operatorname{non}^{*}(\mathcal{I})$

Now we prove the implications of the second row.

$[(\operatorname{non}(\mathcal{I})=|X|)$ or $(\operatorname{add}(\mathcal{I})=\operatorname{cov}(\mathcal{I}))] \Longrightarrow C_{\mathcal{I}}:$ If $\operatorname{non}(\mathcal{I})=|X|$, then the initial segments of any well-ordering $\left\{x_{\alpha}: \alpha<|X|\right\}$ of $X$ belong to $\mathcal{I}$. Next suppose $\operatorname{add}(\mathcal{I})=\operatorname{cov}(\mathcal{I})=\kappa$ and let $X=\cup_{\alpha<\kappa} N_{\alpha}$, where $N_{\alpha} \in \mathcal{I}$ for every $\alpha$. We may assume that the sets $N_{\alpha}$ are pairwise disjoint, and then we can order $X$ in such a way that the elements of $N_{\alpha}$ precede those of $N_{\beta}$ for every $\alpha<\beta$. It is clear that the initial segments of this ordering belong to $\mathcal{I}$. (This argument is well-known; special cases appear in [3, p. 193] and, in the category case, in $[6$, Statement 3, p. 462].)

$C_{\mathcal{I}} \Longrightarrow S_{\mathcal{I}}^{2}$ : This follows by Sierpiński's original argument [9]: If $\prec$ is an ordering of $X$ such that $\{x: x \prec y\} \in \mathcal{I}$ for every $y$, then $H=\{(x, y): x \prec y\}$ satisfies $S_{\mathcal{I}}^{2}$.

$S_{\mathcal{I}}^{2} \Longrightarrow S_{\mathcal{I}}^{2 w}:$ Obvious.

$S_{\mathcal{I}}^{2 w} \Longrightarrow\left[\operatorname{cov}(\mathcal{I}) \leq \operatorname{non}^{*}(\mathcal{I})\right]:$ Suppose that $H$ satisfies $S_{\mathcal{I}}^{2 w}$ and select $E \subset\left\{x: X \backslash H_{x} \in \mathcal{I}\right\}$ such that $E \notin \mathcal{I}$ and $|E| \leq$ non $^{*}(\mathcal{I})$. Then $X=$ $\cup\left\{X \backslash H_{x}: x \in E\right\}$. Indeed, $y \notin X \backslash H_{x}(x \in E)$ would imply $E \subset H^{y}$, contradicting $H^{y} \in \mathcal{I}$. (Cf. [8, p. 306].)

Finally, we turn to the implications of diagonal direction.

$S_{\mathcal{I}}^{2} \Longrightarrow[\operatorname{cov}(\mathcal{I}) \leq \operatorname{non}(\mathcal{I})]:$ Let $E \notin \mathcal{I}$ be such that $|E|=\operatorname{non}(\mathcal{I})$. If $H$ satisfies $S_{\mathcal{I}}^{2}$, then $X=\cup\left\{X \backslash H_{x}: x \in E\right\}$. Indeed, $y \notin X \backslash H_{x}(x \in E)$ would imply $E \subset H^{y}$, contradicting $H^{y} \in \mathcal{I}$. (Cf. [3, p. 193].) 
$[\operatorname{cov}(\mathcal{I}) \leq \operatorname{non}(\mathcal{I})] \Longrightarrow D_{\mathcal{I}}:$ We shall say that the sets $A, B \subset X$ are congruent, if $B=A+x$ for some $x \in X$. First we show that there is a partition $X=X_{1} \cup \ldots \cup X_{k}$ such that $k \geq 2$ is finite and the sets $X_{1}, \ldots, X_{k}$ are congruent to each other. Let $x \neq 0$ be a fixed element of $X$, and let $H$ be a set containing exactly one element of each coset of the group generated by $x$. Then the sets $H+n x(0 \leq n<N)$ constitute a partition of $X$ into congruent subsets, where $N$ denotes the order of $x$. If $N$ is finite, then this is the partition we were looking for. If $N$ is infinite, then we put $X_{1}=\bigcup_{i=0}^{\infty}(H+2 i)$ and $X_{2}=\bigcup_{i=0}^{\infty}(H+(2 i+1))$, and obtain the partition $X=X_{1} \cup X_{2}$.

By the invariance of $\mathcal{I}$ we have $\operatorname{non}\left(\mathcal{I} \mid X_{1}\right)=\ldots=\operatorname{non}\left(\mathcal{I} \mid X_{k}\right)$. This easily implies $\operatorname{non}\left(\mathcal{I} \mid X_{i}\right)=\operatorname{non}(\mathcal{I})$ for every $i=1, \ldots, k$. Similarly, $\operatorname{cov}\left(\mathcal{I} \mid X_{i}\right)=$ $\operatorname{cov}(\mathcal{I})(i=1, \ldots, k)$. Let $A=X_{1}$ and $B=X_{2} \cup \ldots \cup X_{k}$; then we have $\operatorname{cov}(\mathcal{I} \mid A)=\operatorname{cov}(\mathcal{I}) \leq \operatorname{non}(\mathcal{I})=\operatorname{non}(\mathcal{I} \mid B)$ and $\operatorname{cov}(\mathcal{I} \mid B)=\operatorname{cov}(\mathcal{I}) \leq \operatorname{non}(\mathcal{I})=$ $\operatorname{non}(\mathcal{I} \mid A)$.

The two remaining implications follow by transitivity.

\section{Remarks.}

1. It is easy to check that if $X$ is countable, then each statement of Diagram 2 is true except perhaps $G_{\mathcal{I}}, S_{\mathcal{I}}^{1 s}$, and $S_{\mathcal{I}}^{1}$. The statement $G_{\mathcal{I}}$ is false if $X$ is finitely generated or if $X$ is not a torsion group and $\mathcal{I}$ equals the ideal of finite subsets of $X$. Therefore the implication $[\operatorname{non}(\mathcal{I})=|X|] \Longrightarrow G_{\mathcal{I}}$ is false in these cases. The implication $G_{\mathcal{I}} \Longrightarrow S_{\mathcal{I}}^{1 s}$ holds for every group (its proof did not use $|X|>\omega)$. We shall see in the next section that $S_{\mathcal{I}}^{1 s} \Longrightarrow S_{\mathcal{I}}^{1}$ fails in every infinite Abelian group for some ideals.

2. Let $\lambda$ be an infinite cardinal number less than $|X|$, and let $\mathcal{I}=\{H \subset$ $X:|H|<\lambda\}$. Then $\operatorname{cov}(\mathcal{I})=|X|>\operatorname{non}^{*}(\mathcal{I})=\lambda$. Therefore each statement of the diagram is false. In particular, $S_{\mathcal{I}}^{1}$ is false; that is, if $A \subset X,|A| \geq \lambda$ and $|X \backslash A| \geq \lambda$, then there is an $x \in X$ such that $|(A+x) \backslash A| \geq \lambda$. This is Trzeciakiewicz's theorem (see [13] or [4, Theorem 1.7, p. 308]).

3. Suppose $|X| \in\left\{\omega_{1}, \omega_{2}\right\}$, and let $\mathcal{I}$ be an invariant $\sigma$-ideal on $X$. Then we have

$$
\begin{aligned}
{[(\operatorname{non}(\mathcal{I})=|X|) \text { or }(\operatorname{add}(\mathcal{I})=\operatorname{cov}(\mathcal{I}))] } & \Longleftrightarrow C_{\mathcal{I}} \Longleftrightarrow S_{\mathcal{I}}^{2} \\
& \Longleftrightarrow[\operatorname{cov}(\mathcal{I}) \leq \operatorname{non}(\mathcal{I})]
\end{aligned}
$$

Indeed, the implications from left to right are contained in Theorem 2. If $\operatorname{cov}(\mathcal{I}) \leq \operatorname{non}(\mathcal{I})$, then either $\operatorname{non}(\mathcal{I})=|X|$, or $\operatorname{non}(\mathcal{I})=\operatorname{cov}(\mathcal{I})=\omega_{1}$. In the latter case we have $\omega_{1} \leq \operatorname{add}(\mathcal{I}) \leq \operatorname{non}(\mathcal{I})=\omega_{1}$, and then $\operatorname{add}(\mathcal{I})=\omega_{1}=$ $\operatorname{cov}(\mathcal{I})$. (The special case of this observation, when $X=\mathbb{R}$ and $\mathcal{I}$ is the ideal of first category sets, is due to P. Komjáth [6]. See also [3, p. 193].) 
4. In the proof of Theorem 2 the invariance of the ideal was needed in the implications

$$
\begin{aligned}
S_{\mathcal{I}}^{1} \Longrightarrow D_{\mathcal{I}}, S_{\mathcal{I}}^{1 s} & \Longrightarrow S_{\mathcal{I}}^{2},[\operatorname{cov}(\mathcal{I}) \leq \operatorname{non}(\mathcal{I})] \Longrightarrow D_{\mathcal{I}} \\
& \text { and } S_{\mathcal{I}}^{1 s} \Longrightarrow[\operatorname{cov}(\mathcal{I}) \leq \operatorname{non}(\mathcal{I})] .
\end{aligned}
$$

It is easy to check that all the other implications of Diagram 2 hold for any ideal.

\section{On the Implication $S_{\mathcal{I}}^{1 s} \Longrightarrow S_{\mathcal{I}}^{1}$.}

We start with the introduction of two more conditions.

$S_{\mathcal{I}}^{1 w}:$ There are sets $A, B \subset X$ such that $A \notin \mathcal{I}, B \notin \mathcal{I}$, and $(A+h) \cap B \in \mathcal{I}$ for every $h \in X$.

$F_{\mathcal{I}}:$ There exists a proper filter $\mathcal{F}$ on $X$ such that

$$
\mathcal{I}=\{H \subset X: H+x \notin \mathcal{F} \text { for every } x \in X\} .
$$

It is easy to see that $F_{\mathcal{I}}$ is equivalent to the existence of an ultrafilter $\mathcal{F}$ satisfying (1). Indeed, suppose $F_{\mathcal{I}}$, and let $\mathcal{F}^{*}$ be a filter maximal with respect to the properties $\mathcal{F} \subset \mathcal{F}^{*}$ and $\mathcal{F}^{*} \cap \mathcal{I}=\emptyset$. Then $\mathcal{F}^{*}$ is an ultrafilter, since $A \notin \mathcal{F}^{*}$ and $X \backslash A \notin \mathcal{F}^{*}$ would imply, by the maximality of $\mathcal{F}^{*}$, that $A \cap B \in \mathcal{I}$ and $(X \backslash A) \cap C \in \mathcal{I}$ with suitable $B, C \in \mathcal{F}^{*}$. Let $B \cap C=D$. Then $D \in \mathcal{F}^{*}$ and $D \subset(A \cap B) \cup((X \backslash A) \cap C) \in \mathcal{I}$, contradicting $\mathcal{F}^{*} \cap \mathcal{I}=\emptyset$. Since $\mathcal{F} \subset \mathcal{F}^{*}$, (1) remains true when $\mathcal{F}$ is replaced by $\mathcal{F}^{*}$.

We shall also need the converse: If $\mathcal{F}$ is an ultrafilter (not containing $\emptyset$ ) and $\mathcal{I}$ is defined by (1), then $\mathcal{I}$ is an invariant ideal satisfying $F_{\mathcal{I}}$. Or, in an equivalent form, if $\mathcal{I}_{0}$ is a prime ideal, then

$$
\mathcal{I}=\left\{H \subset X: H+x \in \mathcal{I}_{0} \text { for every } x \in X\right\}
$$

is an invariant ideal satisfying $F_{\mathcal{I}}$.

Proposition 3. For every invariant ideal $\mathcal{I}$ we have $S_{\mathcal{I}}^{1} \Longrightarrow S_{\mathcal{I}}^{1 w} \Longrightarrow \neg F_{\mathcal{I}}$.

Proof. The first implication is trivial. To prove the second, suppose that $S_{\mathcal{I}}^{1 w}$ and $F_{\mathcal{I}}$ are both true. Let $\mathcal{F}$ be a filter as in $F_{\mathcal{I}}$, and suppose that $A, B \subset X$ are such that $A \notin \mathcal{I}, B \notin \mathcal{I}$, and $(A+h) \cap B \in \mathcal{I}$ for every $h \in X$. Then there are elements $x, y \in X$ with $A+x, B+y \in \mathcal{F}$. Since $\mathcal{F}$ is a filter, this implies 
$(A+x) \cap(B+y) \in \mathcal{F}$. Therefore $(A+x-y) \cap B \notin \mathcal{I}$, since translating this set by $y$ we obtain an element of $\mathcal{F}$. This contradicts the choice of $A$ and $B$.

Our next aim is to show that the conditions implying $S_{\mathcal{I}}^{1 s} \Longrightarrow S_{\mathcal{I}}^{1}$ are necessary.

Theorem 4. For every infinite Abelian group $X$ there exists an invariant ideal $\mathcal{I}$ on $X$ such that $S_{\mathcal{I}}^{1 s}$ is true but $S_{\mathcal{I}}^{1}$ is false. If $|X|$ is a measurable cardinal, then there is such a $\sigma$-ideal.

Proof. Our first aim is to construct an invariant ideal $\mathcal{J}$ satisfying $S_{\mathcal{J}}^{1 s}$. If $X$ is uncountable, then we put $\mathcal{J}=\{H \subset X:|H|<|X|\}$. Then $\operatorname{non}(\mathcal{J})=|X|$ and hence, by Theorem $2, S_{\mathcal{J}}^{1 s}$ is true.

Next suppose that $X$ is countably infinite. If $X$ is not finitely generated, then let $\mathcal{J}$ denote the system of all sets $H \subset X$ such that the subgroup generated by $H$ is finitely generated. It is clear that $\mathcal{J}$ is an invariant ideal. Let $\left\{x_{n}\right\}_{n=1}^{\infty}$ be an enumeration of $X$, and let $G_{n}$ be the group generated by $x_{1}, \ldots, x_{n}$. Then $G_{1}, G_{2}, \ldots$ is a chain of groups belonging to $\mathcal{J}$, and thus $G_{\mathcal{J}}$ holds. Therefore, $S_{\mathcal{J}}^{1 s}$ holds, also (see the first remark following Theorem 2).

If $X$ is finitely generated, then $G_{\mathcal{J}}$ is false for every invariant ideal; so we need a different construction. By the fundamental theorem, $X$ is the direct sum of a finite subgroup $K$ and a finitely generated free Abelian group. That is, there are elements $h_{1}, \ldots, h_{d}$ such that every element of $X$ can be written uniquely as $g+n_{1} h_{1}+\ldots+n_{d} h_{d}$, where $g \in K$ and $n_{1}, \ldots, n_{d} \in \mathbb{Z}$. Then $\phi\left(g+n_{1} h_{1}+\ldots+n_{d} h_{d}\right)=n_{1}$ defines a homomorphism mapping $X$ onto $\mathbb{Z}$.

We shall say that a set $A \subset \mathbb{Z}$ has density zero if $\lim _{N \rightarrow \infty}|A \cap[-N, N]| / N=$ 0 . Let $\mathcal{J}$ denote the system of all subsets $H \subset X$ such that $\phi(H)$ has density zero. Then $\mathcal{J}$ is an invariant ideal; we show that it satisfies $S_{\mathcal{J}}^{1 s}$.

For every $x \in X$ we define

$$
f(x)= \begin{cases}0 & \text { if }|\phi(x)| \leq 1 \\ n h_{1} & \text { if } 2^{n-1}<|\phi(x)| \leq 2^{n}(n=1,2, \ldots) .\end{cases}
$$

Then $\phi\left(f^{-1}(\{y\})\right)$ is finite for every $y \in X$; in particular, $f^{-1}(\{y\}) \in$ $\mathcal{J}(y \in X)$. Let $y \in X$ be fixed, and put $H=\{x \in X: f(x+y) \neq f(x)\}$. If $x \in H$, then

$$
\phi(x) \in \bigcup_{k=0}^{\infty}\left(\left[-2^{k}-a,-2^{k}+a\right] \cup\left[2^{k}-a, 2^{k}+a\right]\right),
$$

where $a=|\phi(y)|$. This implies that $\phi(H)$ has density zero. Therefore $H \in \mathcal{J}$, and thus $S_{\mathcal{J}}^{1 s}$ holds. 
We have proved that for every infinite Abelian group $X$ there exists an invariant ideal $\mathcal{J}$ satisfying $S_{\mathcal{J}}^{1 s}$. Then $S_{\mathcal{I}}^{1 s}$ is true for every invariant ideal $\mathcal{I}$ with $\mathcal{J} \subset \mathcal{I}$. Let $\mathcal{I}_{0}$ be a prime ideal containing $\mathcal{J}$, and let $\mathcal{I}$ be defined by (2). Since $\mathcal{J}$ is invariant, $\mathcal{I}$ contains $\mathcal{J}$ and thus $S_{\mathcal{I}}^{1 s}$ holds. Also, $\mathcal{I}$ is an invariant ideal satisfying $F_{\mathcal{I}}$ and thus, by Proposition $3, S_{\mathcal{I}}^{1}$ is false. This completes the proof of the first statement of the Theorem.

If $|X|=\kappa$ is measurable, then there is a prime $\sigma$-ideal $\mathcal{I}_{0}$ containing the ideal $\mathcal{J}=\{H \subset X:|H|<\kappa\}$. Let $\mathcal{I}$ be defined by (2); then $\mathcal{I}$ is an invariant $\sigma$-ideal satisfying $F_{\mathcal{I}}$. By Proposition $3, S_{\mathcal{I}}^{1}$ is false. On the other hand, we have $\operatorname{non}(\mathcal{I})=|X|$, since $\mathcal{J} \subset \mathcal{I}$. Thus, by Theorem $2, S_{\mathcal{I}}^{1 s}$ is true.

We conclude this section by showing that, in general, the only implication between the statements of Diagram 2 and $S_{\mathcal{I}}^{1 w}$ is $S_{\mathcal{I}}^{1} \Longrightarrow S_{\mathcal{I}}^{1 w}$.

Theorem 5. Let $\mathcal{I}$ be an invariant ideal such that

$$
\operatorname{non}^{*}(\mathcal{I})<\operatorname{cov}(\mathcal{I})=\operatorname{cof}(\mathcal{I})
$$

Then $S_{\mathcal{I}}^{1 w}$ is true, but every statement of Diagram 2 is false.

Proof. Condition (3) implies $|X|>\omega$, since otherwise non* $(\mathcal{I})=\operatorname{cov}(\mathcal{I})=$ $\omega$. Therefore, by Theorem 2, each of the statements of Diagram 2 is false. Condition (3) also implies that

$$
|X \backslash E| \geq \operatorname{cof}(\mathcal{I}) \text { for every } E \in \mathcal{I} \text {. }
$$

Indeed, suppose $E \in \mathcal{I}$ and $|X \backslash E|<\operatorname{cof}(\mathcal{I})$. Then $X$ can be covered by less than $\operatorname{cof}(\mathcal{I})$ elements of $\mathcal{I}$; namely, by the singletons contained by $X \backslash E$ and by the set $E$. This, however, contradicts (3).

Now we show that (4) implies $S_{\mathcal{I}}^{1 w}$. Let $\operatorname{cof}(\mathcal{I})=\kappa$, and let $E_{\alpha} \in \mathcal{I}(\alpha<\kappa)$ be such that for every $E \in \mathcal{I}$ there is an $\alpha<\kappa$ with $E \subset E_{\alpha}$. Let $x_{0}=y_{0} \in$ $X \backslash E_{0}$. If $0<\alpha<\kappa$ and $x_{\beta}, y_{\beta}$ have been defined for every $\beta<\alpha$, then the set

$$
H_{\alpha}=X \backslash\left[E_{\alpha} \cup\left\{x_{\beta}+y_{\gamma}-y_{\delta}: \beta, \gamma, \delta<\alpha\right\}\right]
$$

is nonempty by (4). Let $x_{\alpha}$ be an arbitrary element of $H_{\alpha}$. The set

$$
K_{\alpha}=X \backslash\left[E_{\alpha} \cup\left\{y_{\beta}+x_{\gamma}-x_{\delta}: \beta<\alpha, \gamma, \delta \leq \alpha\right\}\right]
$$

is also nonempty; let $y_{\alpha} \in K_{\alpha}$. In this way we have selected $x_{\alpha}$ and $y_{\alpha}$ for every $\alpha<\kappa$. Let $A=\left\{x_{\alpha}: \alpha<\kappa\right\}$ and $B=\left\{y_{\alpha}: \alpha<\kappa\right\}$. Then $A, B \notin \mathcal{I}$ since $A, B \not \subset E_{\alpha}$ for every $\alpha$. On the other hand, $x-y=x^{\prime}-y^{\prime}, x, y \in A, x^{\prime}, y^{\prime} \in B$ imply $x=x^{\prime}$ and $y=y^{\prime}$ by the construction. Therefore $|(A+h) \cap B| \leq 1$ and, consequently, $(A+h) \cap B \in \mathcal{I}$ for every $h \in X$.

A simple example of an ideal satisfying (3) is provided by the system of finite subsets of $\mathbb{R}$. 


\section{On the Implication $S_{\mathcal{I}}^{1} \Longrightarrow S_{\mathcal{I}}^{2 w}$.}

If we remove the statement $\operatorname{cov}(\mathcal{I}) \leq \operatorname{non}(\mathcal{I})$ from Diagram 2, the remaining chart suggests $S_{\mathcal{I}}^{1} \Longrightarrow S_{\mathcal{I}}^{2 w}$. We do not know if this is true in general. In the next theorem we shall prove this implication under some extra conditions.

Theorem 6. Let $\mathcal{I}$ be an invariant ideal. If $\operatorname{add}(\mathcal{I})=\operatorname{non}(\mathcal{I})$ or $\operatorname{cov}(\mathcal{I})=$ $\operatorname{cof}(\mathcal{I})$ holds, then $S_{\mathcal{I}}^{1}$ implies $S_{\mathcal{I}}^{2 w}$.

Proof. We may assume $|X|>\omega$, since otherwise $S_{\mathcal{I}}^{2 w}$ is true. Suppose $S_{\mathcal{I}}^{1}$, let $A$ be as in $S_{\mathcal{I}}^{1}$, and put $B=X \backslash A$. Then $\operatorname{cov}(\mathcal{I} \mid A) \leq \operatorname{non}(\mathcal{I} \mid B)$ and $\operatorname{cov}(\mathcal{I} \mid B) \leq \operatorname{non}(\mathcal{I} \mid A)$ (see the proof of $S_{\mathcal{I}}^{1} \Longrightarrow D_{I}$ in the proof of Theorem 2).

First assume $\operatorname{add}(\mathcal{I})=\operatorname{non}(\mathcal{I})=\kappa . \operatorname{Since} \operatorname{non}(\mathcal{I})=\min (\operatorname{non}(\mathcal{I} \mid A), \operatorname{non}(\mathcal{I} \mid B))$, we may assume, by symmetry, that $\operatorname{non}(\mathcal{I})=\operatorname{non}(\mathcal{I} \mid B)=\kappa$. Then

$$
\kappa=\operatorname{add}(\mathcal{I}) \leq \operatorname{add}(\mathcal{I} \mid A) \leq \operatorname{cov}(\mathcal{I} \mid A) \leq \operatorname{non}(\mathcal{I} \mid B)=\kappa
$$

and thus $\operatorname{add}(\mathcal{I} \mid A)=\operatorname{cov}(\mathcal{I} \mid A)$. Therefore $C_{\mathcal{I} \mid A}$ holds and hence there is an ordering $\prec$ of $A$ such that $\{y \in A: y \prec x\} \in \mathcal{I}$ for every $x \in A$. We put

$$
H=\{(x, y) \in A \times A: x \prec y\} \cup\{(x, y) \in X \times X: x \in A \backslash(A-y)\} .
$$

Then $H^{y} \in \mathcal{I}$ for every $y$. We show that $X \backslash H_{x} \in \mathcal{I}$ for every $x \in A$. Since $A \notin \mathcal{I}$, this will prove $S_{\mathcal{I}}^{2 w}$. Let $x \in A$ be fixed. Then $H_{x}=\{y \in A: x \prec$ $y\} \cup(X \backslash(A-x))$, and $X \backslash H_{x} \subset\{y \in A: y \prec x\} \cup\{x\} \cup[(A-x) \backslash A] \in \mathcal{I}$.

Next assume $\operatorname{cov}(\mathcal{I})=\operatorname{cof}(\mathcal{I})=\kappa \cdot \operatorname{Since} \operatorname{cov}(\mathcal{I})=\max (\operatorname{cov}(\mathcal{I} \mid A), \operatorname{cov}(\mathcal{I} \mid B))$, we may suppose that $\operatorname{cov}(\mathcal{I} \mid A)=\kappa$. Our first aim is to construct a map $f: X \rightarrow B$ such that $f(E) \in \mathcal{I}$ for every $E \in \mathcal{I}$, but $f(A) \notin \mathcal{I}$.

Let $\left\{E_{\alpha}: \alpha<\kappa\right\}$ be a subsystem of $\mathcal{I}$ such that every element of $\mathcal{I}$ can be covered by an $E_{\alpha}$. (Such a subsystem exists by $\operatorname{cof}(\mathcal{I})=\kappa$.) Then there are elements $x_{\alpha}(\alpha<\kappa)$ such that

$$
x_{\alpha} \in A \backslash \bigcup_{\beta<\alpha}\left(E_{\beta} \cup\left\{x_{\beta}\right\}\right)
$$

for every $\alpha<\kappa$. Indeed, let $x_{0} \in A$ be arbitrary. If $0<\alpha<\kappa$ and $x_{\beta}$ has been selected for every $\beta<\alpha$, then, by $\kappa=\operatorname{cov}(\mathcal{I} \mid A)$, the sets $E_{\beta} \cup\left\{x_{\beta}\right\}(\beta<\alpha)$ do not cover $A$, and we can choose a point $x_{\alpha}$ satisfying (5). Now

$$
\kappa=\operatorname{cov}(\mathcal{I} \mid A) \leq \operatorname{non}(\mathcal{I} \mid B) \leq \operatorname{cof}(\mathcal{I} \mid B) \leq \operatorname{cof}(\mathcal{I})=\kappa
$$


implies $\operatorname{non}(\mathcal{I} \mid B)=\kappa$ and, consequently, there are elements $b_{\alpha} \in B$ such that $B_{1}=\left\{b_{\alpha}: \alpha<\kappa\right\} \notin \mathcal{I}$. We define $f\left(x_{\alpha}\right)=b_{\alpha}(\alpha<\kappa)$ and $f(x)=b_{0}$ if $x \notin\left\{x_{\alpha}: \alpha<\kappa\right\}$. Then $f: X \rightarrow B_{1}$ and $f(A)=B_{1} \notin \mathcal{I}$. If $E \in \mathcal{I}$, then there is $\alpha<\kappa$ such that $E \subset E_{\alpha}$, and thus

$$
f(E) \subset f\left(E_{\alpha}\right) \subset\left\{b_{\beta}: \beta \leq \alpha\right\} \in \mathcal{I},
$$

since $\operatorname{non}(\mathcal{I} \mid B)=\kappa$. Now we define

$$
H=\{(x, y): y \in X, x \in f(A \backslash(A-y))\} \cup\{(x, y): y \in X, x \in(A-y) \backslash A\} .
$$

Then $H^{y} \in \mathcal{I}$ for every $y$, since $A \backslash(A-y)$ and $(A-y) \backslash A$ both belong to $\mathcal{I}$. On the other hand, if $x \in B_{1} \backslash\left\{b_{0}\right\}$, then $X \backslash H_{x} \in \mathcal{I}$. Indeed, if $y \notin H_{x}$, then $x^{\prime}=f^{-1}(x) \notin A \backslash(A-y)$ and $x \notin(A-y) \backslash A$. Since $x^{\prime} \in A$ and $x \notin A$, we obtain $x^{\prime} \in A-y$ and $x \notin A-y$; that is, $y \in A-x^{\prime}$ and $y \notin A-x$. Thus we have, for every $x \in B_{1} \backslash\left\{b_{0}\right\}$,

$$
X \backslash H_{x} \subset\left(A-x^{\prime}\right) \backslash(A-x)=\left[\left(A-\left(x^{\prime}-x\right)\right) \backslash A\right]-x \in \mathcal{I} .
$$

Since $B_{1} \backslash\left\{b_{0}\right\} \notin \mathcal{I}$, this proves $S_{\mathcal{I}}^{2 w}$.

\section{The Case $X=\mathbb{R}, \mathcal{I}=\mathcal{N}$.}

Let $\mathcal{N}$ denote the ideal of null sets in $\mathbb{R}$. Then the statements of Diagram 2 are independent of ZFC. Indeed, CH or Martin's axiom implies non $(\mathcal{N})=2^{\omega}$ and then, by Theorem 2, each statement of Diagram 2 is true. On the other hand, in the random real model we have $\operatorname{cov}(\mathcal{N}) \geq \omega_{2}$ and non* $(\mathcal{N})=\omega_{1}$ (see Lemma 8 of [8]), and hence in this model each statement of Diagram 2 is false. We remark that $S_{\mathcal{N}}^{1 w}$ is always true, no matter what the actual values of the cardinal functions of $\mathcal{N}$ are. Indeed, $E \in \mathcal{N}$ implies $|\mathbb{R} \backslash E|=2^{\omega} \geq \operatorname{cof}(\mathcal{N})$ (see [1]), and this implies $S_{\mathcal{N}}^{1 w}$ by the proof of Theorem 5 .

In this section we discuss some statements involving real functions that are equivalent to one of $S_{\mathcal{N}}^{2}, S_{\mathcal{N}}^{2 w}$ or $S_{\mathcal{N}}^{1}$.

It is well-known that $S_{\mathcal{N}}^{2}$ is equivalent to the existence of a map $\phi: \mathbb{R} \rightarrow \mathcal{N}$ such that, for every $x, y \in \mathbb{R}, x \neq y$, either $x \in \phi(y)$ or $y \in \phi(x)$. (Proof. If $\phi$ is such a map, then $H=\{(x, y): x \in \phi(y)\}$ satisfies $S_{\mathcal{N}}^{2}$. In the other direction, if $S_{\mathcal{N}}^{2}$ holds for $H \subset \mathbb{R}^{2}$, then $\phi(x)=\{y:(y, x) \in H$ or $(x, y) \notin H\}$ satisfies the requirements; see [3, pp. 197-198] and [12].) In other words, $S_{\mathcal{N}}^{2}$ is equivalent to the negation of condition $A_{\text {null }}$ as defined in [3]. Therefore, by $\left[3\right.$, p. 198], $S_{\mathcal{N}}^{2}$ is equivalent to the existence of a function $f:[0,1]^{2} \rightarrow[0,1]$ such that $\int_{0}^{1}\left(\int_{0}^{1} f d x\right) d y \neq \int_{0}^{1}\left(\int_{0}^{1} f d y\right) d x$. 
In turn, $S_{\mathcal{N}}^{2 w}$ is equivalent to the existence of a non-measurable function $f: \mathbb{R}^{2} \rightarrow \mathbb{R}$ such that the vertical sections of $f$ are approximately continuous and the horizontal sections of $f$ are measurable [8, Lemma 7, p. 303]. Our next aim is to show that $S_{\mathcal{N}}^{1}$ is related to the difference property of the class of measurable functions.

Let $\Delta_{h}$ denote the difference operator; that is, for $h \in \mathbb{R}$ let $\Delta_{h} f(x)=$ $f(x+h)-f(x)$ for every $f: \mathbb{R} \rightarrow \mathbb{R}$ and $x \in \mathbb{R}$. Now $S_{\mathcal{N}}^{1}$ is equivalent to the existence of a non-measurable function $f: \mathbb{R} \rightarrow \mathbb{R}$ such that $\Delta_{h} f=0$ a.e. for every $h \in \mathbb{R}$. Indeed, if $f$ is such a function, then there is a $c \in \mathbb{R}$ such that $A=\{x: f(x) \leq c\}$ is non-measurable. Since $(A+h) \backslash A \subset\left\{x: \Delta_{-h} f(x) \neq 0\right\}$ is of measure zero for every $h$, the set $A$ satisfies $S_{\mathcal{N}}^{1}$.

On the other hand, if $A$ satisfies $S_{\mathcal{N}}^{1}$, then the symmetric difference of $A$ and $A+h$ is of measure zero for every $h$, since $A \backslash(A+h)=[(A-h) \backslash$ $A]+h$. Therefore, if $f$ denotes the characteristic function of $A$, then $f$ is nonmeasurable and $\Delta_{h} f=0$ a.e. A class of real functions $\mathcal{F} \subset \mathbb{R}^{\mathbb{R}}$ is said to have the difference property if, whenever $f: \mathbb{R} \rightarrow \mathbb{R}$ is such that $\Delta_{h} f \in \mathcal{F}$ for every $h \in \mathbb{R}$, then $f=g+H$, where $g \in \mathcal{F}$ and $H$ is additive; that is, $H$ satisfies Cauchy's functional equation $H(x+y)=H(x)+H(y)$ (see [2]). Let $\mathcal{L}$ denote the family of Lebesgue measurable functions defined on $\mathbb{R}$.

Theorem 7. $\mathcal{L}$ has the difference property if and only if $S_{\mathcal{N}}^{1}$ is false.

Proof. Let $A$ be as in $S_{\mathcal{N}}^{1}$. If $f$ denotes the characteristic function of $A$, then $\Delta_{h} f=0$ a.e. and thus $\Delta_{h} f \in \mathcal{L}$ for every $h$. On the other hand, $f$ cannot be written in the form $g+H$, where $g$ is measurable and $H$ is additive. Indeed, if $f=g+H$ where $g$ is measurable, then $H=f-g$ is bounded on a measurable set of positive measure. If $H$ is additive, then this implies that $H$ is linear and that $f=g+H$ is measurable, a contradiction. Therefore, in this case $\mathcal{L}$ does not have the difference property. (This argument is due to Erdős; see [2, p. 195].)

Next suppose $\neg S_{\mathcal{N}}^{1}$, and let $f: \mathbb{R} \rightarrow \mathbb{R}$ be such that $\Delta_{h} f \in \mathcal{L}$ for every $h \in \mathbb{R}$. Then, by [7, Theorem 3], we have $f=g+s+H$, where $g \in \mathcal{L}, H$ is additive and, for every $h, \Delta_{h} s(x)=0$ a.e. Since $S_{\mathcal{N}}^{1}$ is false, this implies that $s$ is measurable. Then $f=(g+s)+H$ is the sum of a measurable function and an additive function, which proves the difference property of the class $\mathcal{L}$.

Theorem 8. It is consistent with $Z F C$ that $\mathcal{L}$ has the difference property.

Proof. As we mentioned before, in the random real model $S_{\mathcal{N}}^{1}$ is false and, consequently, in this model $\mathcal{L}$ has the difference property.

We conclude with some remarks concerning the category analogues of the results above. Let $\mathcal{M}$ denote the ideal of meager subsets (sets of first category) 
of $\mathbb{R}$. The statements of Diagram 2 for $\mathcal{I}=\mathcal{M}$ are, again, independent of ZFC. Namely, under CH or Martin's axiom we have $\operatorname{non}(\mathcal{M})=2^{\omega}$ and then, by Theorem 2, each statement of Diagram 2 is true. On the other hand, by a theorem of Komjáth [5] it is consistent with ZFC that $\operatorname{cov}(\mathcal{M}) \geq \omega_{2}$ and $\operatorname{non}^{*}(\mathcal{M})=\omega_{1}$ and then, in the relevant model each statement of Diagram 2 is false.

Let $\mathcal{B}$ denote the set of functions $f: \mathbb{R} \rightarrow \mathbb{R}$ having the Baire property. It is easy to see that $S_{\mathcal{M}}^{1}$ is equivalent to the existence of a function $f: \mathbb{R} \rightarrow$ $\mathbb{R}, f \notin \mathcal{B}$ such that $\left\{x: \Delta_{h} f(x) \neq 0\right\} \in \mathcal{M}$ for every $h \in \mathbb{R}$. Also, if $S_{\mathcal{M}}^{1}$ is true, then the class $\mathcal{B}$ does not have the difference property. (The proofs of these statements are analogous to those for $\mathcal{I}=\mathcal{N}$.) We do not know whether the difference property of $\mathcal{B}$ is actually equivalent to $\neg S_{\mathcal{M}}^{1}$ or not. The proof of Theorem 7 would certainly work in the category case if the following statement (the analogue of [7, Theorem 3]) were true: If $f: \mathbb{R} \rightarrow \mathbb{R}$ is such that $\Delta_{h} f \in \mathcal{B}$ for every $h \in \mathbb{R}$, then $f=g+s+H$, where $g \in \mathcal{B}, H$ is additive and, for every $h,\left\{x: \Delta_{h} s(x) \neq 0\right\} \in \mathcal{M}$. However, it is not known whether this statement is true or not.

\section{References}

[1] A. Blass, Combinatorial cardinal characteristics of the continuum, Handbook of Set Theory, (editors: M. Foreman, A. Kanamori and M. Magidor). Springer, 1998.

[2] N. G. de Bruijn, Functions whose differences belong to a given class, Nieuw Arch. Wisk. 23 (1951), 194-218.

[3] C. Freiling, Axioms of symmetry: throwing darts at the real number line, J. Symb. Logic 51 (1986), 190-200.

[4] P. Komjáth, Set theoretic constructions in Euclidean spaces, New trends in discrete and computational geometry (editor: J. Pach). Springer, 1993, 303-325.

[5] P. Komjáth, Some remarks on second category sets, Colloq. Math. 66 (1993), 57-62.

[6] P. Komjáth, A note on set mappings with meager images, Studia Sci. Math. Hungar., 30 (1995), 461-467.

[7] M. Laczkovich, Functions with measurable differences, Acta Math. Hungar., 35 (1980), 217-235. 
[8] M. Laczkovich and A. W. Miller, Measurability of functions with approximately continuous vertical sections and measurable horizontal sections, Colloq. Math. 69 (1995), 299-308.

[9] W. Sierpiński, Sur un théorème équivalent à l'hypothèse du continu, Bull. Intern. Acad. Sci. Cracovie, A 1919, 1-3.

[10] W. Sierpiński, Sur les translations des ensembles linéaires, Fund. Math. 19 (1932), 22-28.

[11] W. Sierpiński: Hypothèse du Continu. Second Edition, Chelsea, New York, 1956.

[12] J. C. Simms, Sierpiński's theorem, Simon Stevin, 65 (1991), 69-163.

[13] L. Trzeciakiewicz, Remarque sur les translations des ensembles linéaires, C. R. Soc. Sci. Lettres Varsovie C1. III. 25 (1933), 63-65. 